\title{
Prevalência de mordida aberta anterior e protrusão dentária em pré-escolares da cidade do Recife (PE, Brasil)
}

\author{
Prevalence of anterior open bite and overjet preschoolers \\ in the city of Recife (PE, Brazil)
}

AnaFlávia Granville-Garcia ${ }^{1}$

Jainara M aria Soares Ferreira ${ }^{2}$

Valdenice Aparecida de M enezes ${ }^{2}$

${ }^{1}$ Departamento de Odontologiada Universidade Estadual da Paraíba. R. Juvêncio Arruda, s/no, Bodocongó. 58429-600 Campina Grande PB. anaflaviagg@hotmail.com

${ }^{2}$ Faculdade de 0 dontologia, Universidade do Estado de Pernambuco.
Abstract The objective of this work was to verify the prevalence of malocclusions (anterior open bite, overjet) and its association with age, gender and type of school with a sample of 2,651 preschool children in the city of Recife, PE, Brazil. The children were seated in school chairs in the room of the day care/school for the clinical exam, children aging two years or less were assisted in the system knee-knee, through natural and artificial illumination. The dental protrusion was verified when the overjet was larger than $3 \mathrm{~mm}$ through periodontal probe in millimeters. The presence of anterior open bite was detected when there was no contact with the anterior teeth and the posterior ones stayed in occlusion. $t$ was also registered in clinical record a combination of the two malocclusions types, in other words, open bite and of dental protrusion. The data were analyzed at Qui-square and the association among the events was the odds ratio. The prevalence of protrusion was $66.1 \%$ and previous open bite $19.8 \%$. There was association among this malocclusions, age and type of school, however there was not significant statistical association between malocclusion and gender. It was concluded that the prevalence of malocclusion in preschoolers was high and it was associated to the age and school type. Key words Cross-sectional studies, Deciduous teeth, M alocclusion
Resumo 0 objetivo deste trabalho foi verificar a prevalência de maloclusões (mordida aberta anterior, protrusão dentária) e sua associação com idade, gênero etipo deescola em 2.651 pré- escolares da cidade do Recife, PE, Brasil. As crianças foram sentadas em cadeiras escolares na sala da própria creche/escola para a execução do exame clínico; os menores de dois anos foram atendidos no sistema perna-perna ou joel ho-joelho, por meio de iluminação natural e artificial. A protrusão dentária foi considerada quando sobressaliência maior que $3 \mathrm{~mm}$, mensurada por meio de sonda periodontal milimetrada. A mordida aberta anterior foi registrada quando não houve contato com os dentes anteriores e os posteriores se mantiveram em oclusão. Foi registrada em ficha clínica a combinação dos dois tipos de maloclusão, ou seja, de mordida aberta e de protrusão dentária. Osdados foram avaliados estatisticamente pelo teste Qui-quadrado ea medida para avaliar a associação entre os eventos foi o odds ratio. A prevalência de protrusão dentária foi $66,1 \%$ e mordida aberta anterior, 19,8\%; houve associação entre as maloclusões, idadee tipo deescola, porém não houveassociação estatisticamentesignificante entre maloclusão e gênero. Conclui-se que a prevalência de maloclusões em pré escolares foi ele vada e esteve associada à idade e tipo de escola. Palavras-chave Estudos transversais, Dente de cíduo, M aloclusão 
Introdução

A maloclusão é considerada uma anomalia de desenvolvimento dentário e/ou dos arcos dentários, que ocasiona problemas de ordem estética/ funcional, tendo como causa frequente as condições funcionais adquiridas, em que o desenvolvimento osteogênico, a hereditariedade e o estado geral da criança são fatores contribuintes para a instalação e/ou agravamento dessa patologia ${ }^{1}$.

A prevalência de maloclusão (mordida aberta anterior e protrusão dentária) em pré-escolares relatada na literatura poder ser considerada elevada no Brasil (Tabela 1).

A literatura associa a maloclusão dentária à idade. Crianças mais novas e mais velhas possuem maior prevalência de mordida aberta anterior e protrusão dentária, respectivamente 3 . Sadakyio et al. ${ }^{8}$ e Thomaz eValença ${ }^{5}$ referem que a protrusão dentária diminui com a idade. Katz e Rosenblatt ${ }^{10} \mathrm{e}$ Thomaz e Valença ${ }^{5}$ observaram redução da prevalência da mordida aberta anterior com 0 aumento da idade. Tomita et al. ${ }^{12} \mathrm{ob}$ servaram decréscimo significativo da incidência de maloclusão com a idade.

No que se refere ao gênero mais acometido por maloclusão, Thomaz eValença ${ }^{5}$, Sousa et al. ${ }^{7}$ eSadakyio et al. ${ }^{8}$ verificaram maior prevalência de mordida aberta anterior e/ou protrusão dentária no gênero feminino quando comparado ao masculino. Emmerich et al. ${ }^{6}$ eThomaz eValença ${ }^{5}$ não observaram associação de protrusão dentária e gênero. Carvalho et al. ${ }^{13}$ observaram tendência de maior frequência de maloclusão no gênero masculino em préescolares.
A associação entre maloclusão enível socioeconômico foi estudada por Calisti et al. ${ }^{14} \mathrm{e}$ Thomaz e Valença5; os autores não observaram associação estatisticamente significante entre essas variáveis. Sousa et al. ${ }^{11}$ verificaram associação entre mordida aberta anterior e préescolares de baixa renda.

Este trabalho visa contribuir com os estudos da maloclusão na dentição decídua, verificando a possível relação entre maloclusão (protrusão dentária, mordida aberta), gênero, idade e tipo de escola em préescolares.

\section{Métodos}

Participaram deste estudo descritivo e analítico crianças na faixa etária de 1 a 5 anos das 84 instituições (creches e pré-escolas) públicas ( $n=38$ ) e particulares $(n=48)$ na cidade do Recife, PE, Brasil. A amostra foi aleatória, estratificada eproporcional ao número de alunos por escola, por meio de sorteio por conglomerados (instituições) das seis regiões político-administrativas da cidade do Recife. 0 tamanho amostral foi baseado na estimativa da prevalência de mordida aberta anterior a partir do estudo de Katz e Rosenblatt ${ }^{10}$, para garantir sua representatividade. A partir de um universo de 38.202 crianças, considerando a prevalênciade $33 \%$, erro máximo aceitável de2,5\% enível de confiança de $99 \%$, calculou-se a amostra em 2.212 crianças, por meio do programa Epi Info 6 . Para considerar eventuais perdas amostrais, este valor foi aumentado em aproximadamente $20 \%$, totalizando 2.651 pré-escolares avaliados.

\begin{tabular}{|c|c|c|c|c|c|}
\hline Autor & Ano & Cidade & Amostra & $\begin{array}{c}\text { Mordida aberta } \\
\text { anterior }(\%)\end{array}$ & $\begin{array}{c}\text { Protrusão } \\
\text { dentária (\%) }\end{array}$ \\
\hline Forte e Bosco ${ }^{2}$ & 2001 & Florianópolis & 223 & 24,8 & - \\
\hline Thomaz et al. ${ }^{3}$ & 2002 & $\begin{array}{c}\text { Aracaju } \\
\text { Bayeux } \\
\text { João Pessoa } \\
\text { Recife }\end{array}$ & 989 & - & 36,1 \\
\hline Chevitarese et al. ${ }^{4}$ & 2002 & Rio de Janeiro & 112 & 31,1 & \\
\hline Thomaz \& Valença ${ }^{5}$ & 2005 & São Luís & 1.056 & 15,1 & 27,2 \\
\hline Emmerich et al. ${ }^{6}$ & 2004 & Vitória & 291 & - & 21,3 \\
\hline Sousa et al. ${ }^{7}$ & 2004 & J oão Pessoa & 126 & 13,4 & 12,6 \\
\hline Sadakyio et al. ${ }^{8}$ & 2004 & Piracicaba & 243 & 40,8 & 11,5 \\
\hline Katz et al. ${ }^{9}$ & 2004 & Recife & 330 & 36,4 & - \\
\hline Katz \& Rosenblatt ${ }^{10}$ & 2005 & Recife & 305 & 33,0 & - \\
\hline Sousa et al. ${ }^{11}$ & 2007 & N atal & 366 & 20,6 & - \\
\hline
\end{tabular}


Todos os exames foram realizados pela própria pesquisadora, previamentecalibrada, eduas auxiliares treinadas foram responsáveis pelos registros de dados nas fichas clínicas, colaborando também durante 0 exame.

As crianças ficaram sentadas em cadeiras escolares para a execução dos exames, tendo sido estes realizados em uma sala da própria creche/ escola, com iluminação natural e artificial (lâmpadas). Os participantes menores de dois anos de idade foram atendidos no sistema perna-perna ou joelho-joelho, no qual a criança fica com a cabeça sobre o colo da examinadora, e com 0 tronco e as pernas sobre o colo da auxiliar.

Foram utilizados para realização do exame clínico espelho bucal $n^{\circ} 3$, pinça para algodão e sonda periodontal, esta última para a realização da mensuração da relação horizontal entre os incisivos. Registrou-se na ficha a ausência ou a presença de protrusão dentária sobressaliência superior a $3 \mathrm{~mm}$, conforme Barnet ${ }^{15} \mathrm{e}$ Oliveira et al. ${ }^{16}$. Nas situações em que não houve contato com os dentes anteriores e os posteriores semantiveram em oclusão, foi detectada a presença de mordida aberta ${ }^{16,17}$. Também foi registrado em ficha clínica quando havia combinação dos dois tipos de maloclusão, ou seja, de mordida aberta e de protrusão dentária.

Os dados foram trabalhados por meio de estatística descritiva (números absolutos e percentuais) einferencial (teste Qui-quadrado, teste exato deFisher eíndiceestatístico Kappa). A concordância intraexaminador foi de 0,90 , correspondente à boa confiabilidade.

Este projeto foi aprovado pelo ComitêdeÉtica da Universidade de Pernambuco. Também foram encaminhadas cartas de autorização aos pais e/ou responsáveis para a participação das crianças na pesquisa.

\section{Resultados}

$\mathrm{Na}$ Tabela 2, é possível observar que a maioria $(66,1 \%)$ das crianças não apresentava maloclusão e 19,8\% eram portadoras de mordida aberta. Entreos dois tipos de escola, a maior diferença percentual foi registrada para mordida aberta com valor de 5,3\% (22,4\% na escola pública e $17,1 \%$ na escola particular). Com relação à protrusão dentária, observou-se maior ocorrência na escola privada (13,3\%), quando comparada à pública (10,8\%). Foi comprovada diferença fortementesignificativa entreos dois tipos deescola em relação ao tipo de oclusão.

0 tipo de oclusão por idade analisado pode ser observado na Tabela 3. A prevalência de mordida aberta anterior obteve os dois maiores percentuais nas faixas de 3 e 4 anos. A protrusão dentária obteve maior percentual registrado em crianças com 3 anos e o menor entre as de 1 ano. O bservou-se diminuição de prevalência de maloclusão especialmente na faixa etária entre 3 e 5 anos e associação entre a idade e o tipo de oclusão significativa.

A diferença entre os dois tipos de escola em relação ao tipo de oclusão é significativa para as faixas etárias de 3, 4 e 5 anos (Tabela 4).

Em relação ao gênero, é possível verificar prevalência semel hante em relação ao tipo de oclusão, e não se comprova associação significativa entre as duas variáveis (Tabela 5).

\section{Discussão}

A prevalência de protrusão dentária encontrada neste estudo foi de $66,1 \%$ (Tabela 2), sendo considerada elevada quando comparada aos traba-

Tabela 2. Avaliação do tipo de oclusão por tipo de escola.

\begin{tabular}{|c|c|c|c|c|c|c|c|}
\hline \multirow{3}{*}{ Tipo oclusão } & \multicolumn{4}{|c|}{ Tipo de escola } & & & \multirow{3}{*}{ Valor de P } \\
\hline & \multicolumn{2}{|c|}{ Particular } & \multicolumn{2}{|c|}{ Pública } & \multicolumn{2}{|c|}{ Grupo total } & \\
\hline & $\mathrm{N}$ & $\%$ & $\mathrm{~N}$ & $\%$ & $\mathrm{~N}$ & $\%$ & \\
\hline Sem maloclusão & 865 & 65,9 & 887 & 66,3 & 1.752 & 66,1 & $P<0,0001^{*}$ \\
\hline Protrusão & 175 & 13,3 & 145 & 10,8 & 320 & 12,1 & \\
\hline M ordida aberta & 225 & 17,1 & 299 & 22,4 & 524 & 19,8 & \\
\hline M ordida aberta + protrusão & 48 & 3,7 & 7 & 0,5 & 55 & 2,1 & \\
\hline Total & 1.313 & 100,0 & 1.338 & 100,0 & 2.651 & 100,0 & \\
\hline
\end{tabular}

*Diferença significativa no nível de 5,0\%. 
Ihos de Thomaz e Valença ${ }^{5}$, Sousa et al. ${ }^{7}$, Sadakyio et al. ${ }^{8}$, Emmerich et al. ${ }^{6}$ e Thomaz et al. ${ }^{3}$. Já a prevalência de mordida aberta anterior encontrada, 19,8\% (Tabela 2), foi semelhante aos achados de Forte e Bosco ${ }^{2}$, Thomaz e Valença ${ }^{5} \mathrm{e}$ Sousa et al. ${ }^{11}$ e baixa quando comparada aos estudos de Chevitarese et al. ${ }^{4}$, Katz et al. ${ }^{9}$ e Katz e Rosenblatt ${ }^{10}$.

\begin{tabular}{|c|c|c|c|c|c|c|c|c|c|c|c|}
\hline \multirow{3}{*}{$\begin{array}{l}\text { Faixa etária } \\
\text { (em anos) }\end{array}$} & \multicolumn{8}{|c|}{ Oclusão } & & & \multirow{3}{*}{ Valor de P } \\
\hline & \multicolumn{2}{|c|}{$\begin{array}{c}\text { Sem } \\
\text { malocusão }\end{array}$} & \multicolumn{2}{|c|}{ Protrusão } & \multicolumn{2}{|c|}{$\begin{array}{l}\text { Mordida } \\
\text { aberta }\end{array}$} & \multicolumn{2}{|c|}{$\begin{array}{l}\text { Mordida aberta } \\
\text { + protrusão }\end{array}$} & \multicolumn{2}{|c|}{ Total } & \\
\hline & $\mathrm{N}$ & $\%$ & $\mathrm{~N}$ & $\%$ & $\mathrm{~N}$ & $\%$ & $\mathrm{~N}$ & $\%$ & $\mathrm{~N}$ & $\%$ & \\
\hline 1 & 164 & 71,6 & 32 & 14,0 & 32 & 14,0 & 1 & 0,4 & 229 & 100,0 & $P<0,0001^{*}$ \\
\hline 2 & 232 & 55,8 & 70 & 16,8 & 104 & 25,0 & 10 & 2,4 & 416 & 100,0 & \\
\hline 3 & 335 & 56,9 & 76 & 12,9 & 161 & 27,3 & 17 & 2,9 & 589 & 100,0 & \\
\hline 4 & 425 & 64,6 & 72 & 10,9 & 142 & 21,6 & 19 & 2,9 & 658 & 100,0 & \\
\hline 5 & 596 & 78,5 & 70 & 9,2 & 85 & 11,2 & 8 & 1,0 & 759 & 100,0 & \\
\hline Total & 1.752 & 66,1 & 320 & 12,1 & 524 & 19,8 & 55 & 2,1 & 2.651 & 100,0 & \\
\hline
\end{tabular}

*Associação significativa no nível de 5,0\%.

Tabela 4. Avaliação da idade das crianças (em anos) segundo o tipo de oclusão por tipo de escola.

\begin{tabular}{|c|c|c|c|c|c|c|c|c|}
\hline \multirow{3}{*}{$\begin{array}{l}\text { Faixa etária } \\
\text { (em anos) }\end{array}$} & \multirow{3}{*}{ Tipo oclusão } & \multicolumn{4}{|c|}{ Tipo de escola } & & & \multirow{3}{*}{ Valor de P } \\
\hline & & \multicolumn{2}{|c|}{ Particular } & \multicolumn{2}{|c|}{ Pública } & \multicolumn{2}{|c|}{ Grupo total } & \\
\hline & & $\mathrm{N}$ & $\%$ & $\mathrm{~N}$ & $\%$ & $\mathrm{~N}$ & $\%$ & \\
\hline \multirow[t]{4}{*}{1} & Sem maloclusão & 46 & 75,4 & 118 & 70,2 & 164 & 71,6 & \multirow{5}{*}{$P^{* *}=0,2972$} \\
\hline & Protrusão & 8 & 13,1 & 24 & 14,3 & 32 & 14,0 & \\
\hline & M ordida aberta & 6 & 9,8 & 26 & 15,5 & 32 & 14,0 & \\
\hline & M ordida aberta + protrusão & 1 & 1,6 & - & - & 1 & 0,4 & \\
\hline Total & & 61 & 100,0 & 168 & 100,0 & 229 & 100,0 & \\
\hline \multirow[t]{4}{*}{2} & Sem maloclusão & 83 & 54,6 & 149 & 56,4 & 232 & 55,8 & \multirow{5}{*}{$P=0,1672$} \\
\hline & Protrusão & 26 & 17,1 & 44 & 16,7 & 70 & 16,8 & \\
\hline & M ordida aberta & 36 & 23,7 & 68 & 25,8 & 104 & 25,0 & \\
\hline & M ordida aberta + protrusão & 7 & 4,6 & 3 & 1,1 & 10 & 2,4 & \\
\hline Total & & 152 & 100,0 & 264 & 100,0 & 416 & 100,0 & \\
\hline \multirow[t]{4}{*}{3} & Sem maloclusão & 157 & 53,6 & 178 & 60,1 & 335 & 56,9 & \multirow[t]{5}{*}{$P=0,0038^{*}$} \\
\hline & Protrusão & 44 & 15,0 & 32 & 10,8 & 76 & 12,9 & \\
\hline & M ordida aberta & 77 & 26,3 & 84 & 28,4 & 161 & 27,3 & \\
\hline & M ordida aberta + protrusão & 15 & 5,1 & 2 & 0,7 & 17 & 2,9 & \\
\hline Total & & 293 & 100,0 & 296 & 100,0 & 589 & 100,0 & \\
\hline \multirow[t]{4}{*}{4} & Sem maloclusão & 241 & 64,1 & 184 & 65,3 & 425 & 64,6 & \multirow[t]{5}{*}{$P<0,0001^{*}$} \\
\hline & Protrusão & 44 & 11,7 & 28 & 9,9 & 72 & 10,9 & \\
\hline & M ordida aberta & 72 & 19,2 & 70 & 24,8 & 142 & 21,6 & \\
\hline & M ordida aberta + protrusão & 19 & 5,1 & - & - & 19 & 2,9 & \\
\hline Total & & 376 & 100,0 & 282 & 100,0 & 658 & 100,0 & \\
\hline \multirow[t]{4}{*}{5} & Sem maloclusão & 338 & 78,4 & 258 & 78,7 & 596 & 78,5 & \multirow[t]{5}{*}{$P<0,0001^{*}$} \\
\hline & Protrusão & 53 & 12,3 & 17 & 5,6 & 70 & 9,2 & \\
\hline & M ordida aberta & 34 & 7,9 & 51 & 15,5 & 85 & 11,2 & \\
\hline & M ordida aberta + protrusão & 6 & 1,4 & 2 & 0,6 & 8 & 1,1 & \\
\hline Total & & 431 & 100,0 & 328 & 100,0 & 759 & 100,0 & \\
\hline
\end{tabular}

*Diferença significativa no nível de 5,0\%; **Através do teste exato de Fisher. 


\begin{tabular}{|c|c|c|c|c|c|c|c|c|c|c|c|}
\hline \multirow{3}{*}{ Sexo } & \multicolumn{8}{|c|}{ Oclusão } & & & \multirow{3}{*}{ Valor de } \\
\hline & \multicolumn{2}{|c|}{$\begin{array}{c}\text { Sem } \\
\text { malocusão }\end{array}$} & \multicolumn{2}{|c|}{ Protrusão } & \multicolumn{2}{|c|}{$\begin{array}{c}\text { Mordida } \\
\text { aberta }\end{array}$} & \multicolumn{2}{|c|}{$\begin{array}{l}\text { Mordida aberta } \\
\text { + protrusão }\end{array}$} & \multicolumn{2}{|c|}{ Total } & \\
\hline & $\mathrm{N}$ & $\%$ & $\mathrm{~N}$ & $\%$ & $\mathrm{~N}$ & $\%$ & $\mathrm{~N}$ & $\%$ & $\mathrm{~N}$ & $\%$ & \\
\hline Masculino & 926 & 66,6 & 171 & 12,3 & 266 & 19,1 & 27 & 1,9 & 1.390 & 100,0 & $P=0,7805$ \\
\hline Feminino & 826 & 65,5 & 149 & 11,8 & 258 & 20,5 & 28 & 2,2 & 1.261 & 100,0 & \\
\hline Total & 1.752 & 66,1 & 320 & 12,1 & 524 & 19,8 & 55 & 2,1 & 2.651 & 100,0 & \\
\hline
\end{tabular}

Com relação à associação entre idade e maloclusão (Tabela 3), observou-se diminuição de sua prevalência, especialmentena faixa etária entre 3 e 5 anos. Esses achados corroboram os estudos de Sadakyio et al. ${ }^{8}$, Thomaz eValença ${ }^{5}$, Katz e Rosenblatt ${ }^{10}$ e Tomita et al. ${ }^{12}$.

Verificou-se associação significativa entrepresença da mordida aberta anterior e préescolares pertencentes à rede pública, especialmente para idades de 3 a 5 anos (Tabela 4). Esses resultados são divergentes do estudo de Sousa et al. ${ }^{11}$, que não observou associação significanteentreo tipo de estabelecimento de ensino e a presença dessa patologia.

Já a protrusão dentária foi mais frequente em escolas privadas nessa mesma faixa etária (Tabela 4). Resultado semel hante ocorreu no estudo de Thomaz e Valença ${ }^{5}$ em escolas da zona urbana.

Analisando-se a presença de maloclusão dentária quanto ao gênero (Tabela 5), não foi comprovada associação significativa entre as duas variáveis. No que se refere à protrusão dentária, nossos resultados foram confirmados pelos estudos de Emmerich et al. ${ }^{6}$ e Thomaz e Valença ${ }^{5}$, mas divergem dos estudos de Thomaz e Valença ${ }^{5}$, Sousa et al. ${ }^{7}$ e Sadakyio et al. ${ }^{8}$ no queserefere à sua associação à mordida aberta anterior.

Seria válida a investigação, nessa população, da influência de fatores extrínsecos associadosà mal oclusão dentária, como os hábitos orais deletérios, pois esta frequentemente vem sendo associada à presença de hábitos de sucção dechupeta e de dedo 2,6,9-11,18,19, que interagem com 0 padrão facial do indivíduo desencadeando ou intensificando o desequilíbrio dentofacial.
Os resultados desta pesquisa apontam a maloclusão dentária como problema de saúde pública na cidade do Recife, especialmente em crianças de tenra idade. Esse fato causa um impacto negativo significativo na qualidade de vida das crianças e de seus familiares ${ }^{20}$, devido às implicações fisiológicas e sociais decorrentes dessa desordem.

Estelevantamento epidemiológico contribuiu para o conhecimento de oclusopatias em préescolares da cidade do Recife, no sentido de incorporar novos programas de atenção à saúde bucal por parte dos gestores de saúde, colaborando para o planejamento e a alocação de recursos em políticas públicas de saúde.

Algumas reflexões podem ser elaboradas diante dos resultados obtidos, como a importância do desenvolvimento de estratégicas coletivas baseadas na prevenção de maloclusões dentárias em pré-escolares, da capacitação dos professores da préescola no controle de seus fatores de risco e da indicação para intervenção ortodôntica precoce por parte dos profissionais de saúde.

\section{Conclusões}

Conclui-se que a prevalência de maloclusões em préescolares da cidade do Recife foi elevada, havendo associação com a idade e o tipo de escola. Os agravos e a desigualdade na distribuição das oclusopatias nessa população podem ser minimizados por meio da integralidade, contemplando prevenção, promoção e tratamento de saúde bucal. 


\section{Colaboradores}

AF Granville-Garcia trabalhou na concepção, coleta e análise de dados e redação; JM S Ferreira trabalhou na análise de dados e redação; VA Menezes, na concepção, metodologia eredação final.

\section{Referências}

1. Nield LS, Stenger JP, Kamat D. Common pediatric dental dilemmas. Clinical Pediatrics 2007; 20(10):1-7.

2. Forte FDS, Bosco VL. Prevalência de mordida aberta anterior e sua relação com hábitos de sucção não nutritiva. Pesq Bras O dontoped Clin Integr 2001; 1(1):38.

3. Thomaz EBAF, Ely MR, Moraes ES, Valença AM G. Prevalência de protrusão dos incisivos superiores, sobremordida profunda, perda prematura de elementos dentários e apinhamento na dentição decídua. J Bras O dontoped O dontol Bebê 2002; 5(26):276282.

4. Chevitarese ABA, Valle DD, Moreira TC. Prevalence of malocclusion in 4-6 year old Brazilian children. Int J Paediatr Dent 2002; 27(1):81-85.

5. Thomaz EBAF, Valença AM G. Prevalência de máoclusão e fatores relacionados à sua recorrência em pré-escolares da cidade de São Luís - MA Brasil. Rev Pós Grad 2005; 12(2):212-221.

6. Emmerich A, Fonseca L, Elias AM, Medeiros UV. Relação entre hábitos bucais, alterações oronasofaringeanas e mal-oclusões em pré-escolares de Vitória, Espírito Santo, Brasil. Cad Saude Publica 2004; 20(3):689-697.

7. Sousa FRN, Taveira GS, Almeida RVD, Padilha WWN. O aleitamento materno e sua relação com hábitos deletérios e maloclusão. Pesq Bras Odontoped Clin Integr 2004; 4(3):211-216.

8. Sadakyio CA, Degan VV, Pignataro Neto G, Puppin Rontani RM. Prevalência de má oclusão em préescolares de Piraciba-SP. Cienc Odontol Bras 2004; 7(2):92-99.

9. Katz CRT, Rosenblatt A, Gondim PPC. Nonnutritive sucking habits in Brazilian children: effects on deciduous dentition and relationship with facial morphology. American Journal of Orthodontics and Dentofacial Orthopedics 2004; 126(1):53-57.

10. Katz CRT, Rosenblatt A. Nonnutritive sucking habits and anterior open bite in Brazilian children: a longitudinal study. Pediatric Dentistry 2005; 27(5):1-5.

11. Sousa RLS, Lima RB, Florêncio Filho C, Lima KC, Diógenes AMN N Prevalência e fatores de risco da mordida aberta anterior na dentadura decídua completa em préescolares de Natal/RN. R Dental Press Ortodont Ortop Facial 2007; 12(2):129-138.
12. Tomita NE, Bijella VT, Franco LJ. The relationship between oral habits and malocclusion in preschool children. Rev Saude Publica 2000; 3(3):299-303.

13. Carvalho JC, Vinker F, Declerck D. Maloclusion, dental injuries and anomalies in the primary dentition of Belgian children. Int J Paediatr Dent 1998; $8(2): 137-141$

14. Castili LJP, Cohen MM, Fales MH. Correlation between malocclusion, oral habits, and socioeconomic level of preschool children. J Dent Res 1960; 39(3):450-454.

15. Barnet EM. Terapia oclusal en odontopediatria. Buenos Aires: Panamericana; 1978.

16. Oliveira LB, Marcenes W, Ardenghi TM, Sheiham $A$, Bönecker $M$. Traumatic dental injuries and associated factors among Brazilian preschool children. Dent Traumatol 2007; 23(2):76-81.

17. Otuyemi OD. Traumatic anterior dental injuries related to incisor overjet and lip competence in 12year-old Nigerian children. Int J Paediatr Dent 1994; $4(2): 81-85$.

18. Martins JCR, Sinimbu CMB, Dinelli TCS, Martins LPM, Raveli DB. Prevalência de má oclusão em pré-escolares de Araraquara: relação da dentição decídua com hábitos e nível socioeconômico. $R$ Dental Press Ortodont Ortop Facial 1998; 3(6):35-43.

19. Cavalcanti AL, Bezerra PKM, M oura C. Aleitamento natural, aleitamento artificial, hábitos de sucção e maloclusões em pré-escolares brasileiros. Rev Salud Pública 2007; 9(2):194-204.

20. Johal $A$, Cheung MY, Marcene $W$. The impact of two different malocclusion traits on quality of life. Br Dent J 2007; 202(2):27.

Artigo apresentado em 19/12/2007

A provado em 01/12/2008

Versão final apresentada em 11/12/2008 